\title{
A Novel Channel Sensing Method for IEEE802.11n MIMO System
}

\author{
Yao Xiaocheng ${ }^{1,2}$, Fang Zhi $^{1}$, Jian Haifang ${ }^{1}$, Shi Yin ${ }^{1}$ \\ 1. Institute of Semiconductors Chinese Academy of Science, Beijing, China \\ 2. Department of Electronic Engineering, Tsinghua University
}

\begin{abstract}
Coexistence and interoperability between $20 \mathrm{MHz}$ and $40 \mathrm{MHz}$ device and modes of operation are addressed in standard IEEE802.11n system. It is mandate to give the channel state in receiving processing, since for $40 \mathrm{MHz}$ device and modes for it should not only fulfill the single $20 \mathrm{MHz}$ signal but also $40 \mathrm{MHz}$ signal receiver requirement. Both energy detection and carrier sense are employed to detecting channel state. In the case of $20 / 40 \mathrm{M}$ mode, the power difference between the two sub-channels is also detected in order to indicate the channel state accurately. The simulation results demonstrate that the performance of the proposed methods is much better than the methods which just taking the first step above.
\end{abstract}

Keywords- MIMO-OFDM, Channel Detection, IEEE802.11n, Carrier Sense, Energy Detection

\section{INTRODUCTION}

As increasing of consumers' demands for high-quality and high throughput multimedia services, IEEE 802.11n wireless LAN (WLAN) standard has been defined to support high speed and high reliable wireless communication. IEEE $802.11 \mathrm{n}$ which is the high throughput amendment to the 802.11 standard specifies a multiple-input and multipleoutput orthogonal frequency division multiplexing (MIMOOFDM) based WLAN systems, and it can support a maximum data rate of 600Mbps at physical (PHY) layer [1].

The combination of MIMO technique with OFDM has been widely adopted in broadband wireless communication system for OFDM has considerable robustness over severe multi-path fading, narrow band interference while MIMO can enhance the capacity of the channel without increasing the power of the transmitter. However, channel detection which is processed in time domain can employ little of these priorities. Incorrect channel detection immediately influences the system performance. Multi-path fading and long delay spread degrades the channel detection performance severely.

In burst wireless communication systems such as IEEE 802.11n WLAN systems, the mechanism of receive and transmit is based on channel state. It is necessary for physical layer to report the channel state to MAC. Several approaches on channel detection have been proposed in literatures $[2,3,4]$.

The rest of the paper is organized as follows. In Section II, we describe IEEE 802.11n system model and present the problem caused by long delay spread as well as cyclic shift delay (CSD) scheme. The proposed channel detection method is presented in Section III, and simulation results are provided in Section IV. Finally, Section V concludes the paper.

\section{System Model Of The IEeE802.11N Mimo-OFdM}

PHY data rates in $802.11 \mathrm{n}$ are significantly improved over $802.11 \mathrm{a}$ and $802.11 \mathrm{~g}$ primarily through the use of spatial multiplexing using MIMO and $40 \mathrm{MHz}$ operation. To take advantage of the much higher data rates provided by these techniques, MAC efficiency is also improved through the use of frame aggregation and enhancements to the block acknowledgment protocol. These features together provide the bulk of the throughput enhancement over the achievable with $802.11 \mathrm{a}$ and $802.11 \mathrm{~g}$. Robustness is improved inherently through the increased spatial diversity provided by the use of multiple antennas. Space-time block coding (STBC) as an option in the PHY further improves robustness, as does fast link adaptation, a mechanism for rapidly tracking changing channel conditions. More robust channel codes are adopted in the form of low density parity check (LDPC) codes. The standard amendment also introduces transmit beam-forming, with both PHY and MAC enhancements to further improve robustness [8].

A number of other enhancements provide further improvements. In the PHY, these include a shorter guard interval, which may be used under certain channel conditions. The PHY also includes a Greenfield preamble, which is shorter than the mandatory mixed format preamble.

Two HT formats are added to IEEE 802.11n: HT-mixed format and HT-greenfield format. The legacy format is named after non-HT format. The PPDU structure is shown in Figure 1.
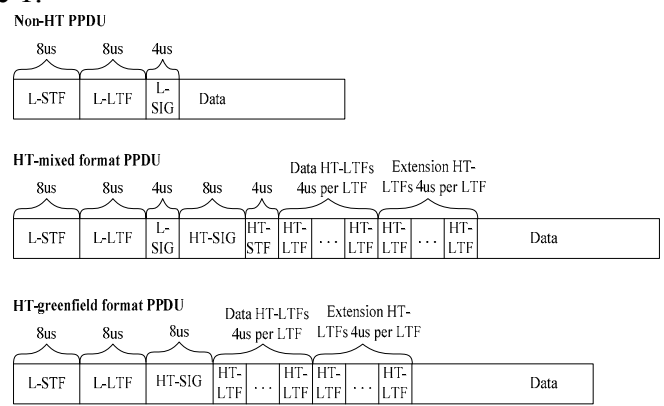

Figure 1. PPDU format in the IEEE 802.11n standard

In order to avoid undesired beam-forming and achieve spatial diversity, CSD scheme is deployed to the preamble for every OFDM symbol. In this scheme, transmitted signals from each TX chain are cyclic shift delayed versions of its original modulated signal. For example, if $T X i$ has a cyclic shift delay of ${ }^{t_{i}}$ the transmitted OFDM symbol from $T X i$ is described as: 


$$
T X_{i}(t)= \begin{cases}x\left(t-t_{i}\right) & 0 \leq t<T_{F F T}+t_{i} \\ x\left(t-t_{i}-T_{F F T}\right) & T_{F F T}+t_{i} \leq t<T_{F F T}\end{cases}
$$

where $\mathrm{x}($.$) is the signal from T X i$ before CSD, $T X i(\mathrm{t})$ is the signal from transmit chain $T X i$ after CSD, and $T_{F F T}$ denotes the total duration of a Fast Fourier Transform (FFT). The values of the cyclic shift is applied to these streams during non-HT portion and the HT portion are specified in Table 21. The received signal at the $R X_{i}$ is the sum of the signals from all TX chains via wireless channel including additive white Gaussian noise (AWGN) which shall be as shown in Equation 2:

$$
R X_{i}(t)=\sum_{j=1}^{N_{T}} \sum_{l=0}^{L-1} h_{j, i}(l) T X_{j}(t-l)+w_{i}(t)
$$

where ${ }^{h_{i i}(l)}$ is the $l$-th sampled channel impulse response (CIR) of the channel between ${ }^{T X_{j}}$ and ${ }^{R X_{i}}, \mathrm{~L}$ is the length of CIR, and $w_{i}(\mathrm{t})$ is the AWGN term.

\begin{tabular}{|c|c|c|c|c|}
\hline \multicolumn{5}{|l|}{$T_{C S}^{i_{T X}}$} \\
\hline $\begin{array}{c}\text { Number of } \\
\text { transmit } \\
\text { chains }\end{array}$ & $\begin{array}{l}\text { Cyclic shift } \\
\text { for transmit } \\
\text { chain } 1 \text { (ns) }\end{array}$ & $\begin{array}{l}\text { Cyclic shift } \\
\text { for transmit } \\
\text { chain } 2 \text { (ns) }\end{array}$ & $\begin{array}{c}\text { Cyclic } \\
\text { shift for } \\
\text { transmit } \\
\text { chain } 3 \\
\text { (ns) }\end{array}$ & $\begin{array}{c}\text { Cyclic } \\
\text { shift for } \\
\text { transmit } \\
\text { chain } 4 \\
\text { (ns) }\end{array}$ \\
\hline 1 & $0 / 0$ & 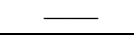 & $\square$ & $\square$ \\
\hline 2 & $0 / 0$ & $-200 /-400$ & $\bar{\square}$ & $\square$ \\
\hline 3 & $0 / 0$ & $-100 /-400$ & $-200 /-200$ & - \\
\hline 4 & $0 / 0$ & $-50 /-400$ & $-100 /-200$ & $-150 /-600$ \\
\hline
\end{tabular}

\section{TABLE I. CYCLIC SHIFT FOR NON-HT PORTION OF PACKET}

We consider a system with $N_{T}$ transmit antennas and $N_{R}$ receive antennas for the description of the impact of delay spreading channel and CSD. The received signal at the $R X_{i}$ is the sum of all the signal which are transmitted using CSD scheme by multiple transmit antennas through delay spreading multipath fading channels, as in Eq. (3):

$$
y_{R X_{i}}(t)=\sum_{j=1}^{N_{T X}} h_{R X_{i, j}}(t) * x_{j}(t)
$$

where $*$ is a convolution operation, $h_{R X_{j}}(t)$ is the impulse response of the channel between ${ }^{T X_{j}}$ and ${ }^{R X_{i}}$. Under long delay spread multipath channels and using CSD scheme, the peak to average ratio of cross-correlation becomes lower and it is hard to detect whether the coming signal is the required or not. The auto-correlation of the ${ }^{R X_{i}}$ with time lag of Ls (samples in 0.8us for OFDM) which is the shortest duration of a short training field signal, as shown in Eq. (4):

$$
A(n)=\sum_{k=1}^{L s} y(n+k) y^{*}(n+k+L s)
$$

where $A(n)$ is the auto-correlation output and $y(n)$ is the $n$th sampled data of the received data $y_{R X_{i}}(t)$ with physical layer impairments including Tx and Rx phase noise, power amplifier (PA) non-linearity and carrier frequency and clock symbol offset. In Figure 2, the auto-correlation output of the preamble part of IEEE $802.11 \mathrm{n}$ frame is shown. We take $2 T X \times 1 R X$ for example in which the TX1 has a CSD value of 0 and TX2 has a negative CSD of ${ }^{T_{c S}^{i s s}}(-400 \mathrm{~ns}$ in HT-greenfield format and -200ns in Non-HT and HT-mixed format as shown in table 2-1) and the rectangular is the autocorrelation window.

\section{Proposed Channel Detection Methoed}

Channel bonding, also known as $40 \mathrm{MHz}$, allows $802.11 \mathrm{n}$ devices to simultaneously use two separate non-overlapping channels to transmit data. So it is necessary to sense the both channel and report the channel state to Medium Access Control (MAC) layer. Channel bonding increases the amount of data that can be transmitted, or decreases the communication delay which is important for delay-sensitive multimedia applications by doubling the raw PHY data rate compared to single channel communication.

The 802.11 Distributed Coordination Function (DCF) relies on sensing of the wireless channel. A station with a new packet to transmit senses the channel activity. If the channel is sensed idle for a period of time equal to a distributed inter-frame space (DIF), the station transmits. On the other hand if the channel is sensed busy, the station persists to monitor the channel until it is measured idle for more than a DIFS. A station should sense the channel activity before AGC when in receive mode. Channel sensing is the essential part of the $802.11 \mathrm{n}$ system which is the basis of transmit and the receive switching. We proposed a novel channel sensing method which is divided into three steps. In the first step, when an AP (access point) or a non-AP STA switches to $20 \mathrm{MHz} / 40 \mathrm{MHz}$ mode [6], the received data is divided into two parts: upper-band and lower-band data defined in $802.11 \mathrm{n}$ standard by employing Hilbert filter. In the second step, both energy detection and carrier sensing are used to sense the data provided in the first step. In the third step, the channel state is reported by taking the result of both energy detection and carrier sensing into consideration.

The Hilbert filter can be used to relate the real and imaginary components of a network function specified in the frequency domain, or to relate a pair of waveforms in the time domain [8]. Thus we employ an impulse response digital filter (which is called Hilbert filter for short in the paper) to realize the discrete Hilbert transform. An impulse response digital filter with a unit-sample response is defined, as in Eq. (5).

$$
h_{N}(n)= \begin{cases}\frac{2}{N} \cot \left(\frac{\pi}{N} n\right) & n= \pm 1, \pm 3, \ldots \pm\left(\frac{N}{2}-1\right) \\ 0 & n=0, \pm 2, \pm 4, \ldots, \pm\left(\frac{N}{2}-2\right)\end{cases}
$$

In this case we have

$h_{N}(n)=-h_{N}(-n)$

The frequency response of the filter for $0 \leq f \leq 1 / 2$ may be obtained by evaluating the discrete Fourier transform as described in [6], as in Eq. (7)

$$
H_{N}(k)= \begin{cases}0 & k=-N / 2 \\ +j & k=-N / 2+1,-N / 2+2, \ldots,-1 \\ 0 & k=0 \\ -j & k=1,2, \ldots N / 2-1\end{cases}
$$

It is clear that the useful frequency band inside which the magnitude characteristic of the filter remains within a percentage points of unity improves markedly with increase the order $\mathrm{N}$ of the filter since the pass-band is no less than $[1 / \mathrm{N} 1 / 2-1 / \mathrm{N}]$ in the positive frequency part. Considering the bandwidth of the effective data (non-HT short training field 
as defined in IEEE 802.11n standard) for channel sensing, $\mathrm{N}=16$ is large enough for dividing the channel.

The upper-band data can be denoted as in Eq. (8).

$$
r_{\text {upperband }}(k)=r(k)+j * \sum_{i=-N / 2+1}^{N / 2-1} h_{N}(i) * r(k-i)
$$

where $r_{\text {uppertand }}(k)$ is the upper-band sequence, $\mathrm{r}()$ is the received data sequence in $20 \mathrm{MHz} / 40 \mathrm{MHz}$ mode, $h_{N}()$ is the impulse response of the Hilbert filter. The lower-band data can be denoted in the similar way as in Eq. (9)

$$
r_{\text {lowerband }}(k)=r(k)-j * \sum_{i=-N / 2+1}^{N / 2-1} h_{N}(k) * r(k-i)
$$

When the device is switch to $20 \mathrm{MHz} / 40 \mathrm{MHz}$ mode, the receiver occupies two separate non-overlapped channels. The method proposed above can be used to divide the two separate channels when the device in $20 \mathrm{MHz} / 40 \mathrm{MHz}$ mode.

Energy detection and carrier sensing are the common methods which are always employed to sense channel. However many papers and standards mention channel sensing, little give detail description and enough simulation results. In the paper, we proposed a novel channel sensing method which employed both energy detection (ED) and carrier sensing $(\mathrm{CS})$. When the coming data is $20 \mathrm{MHz}$ only or two divided channel (lower-band data and upper-band data), we simultaneously sense carrier and detect the channel energy. In 802.11n MIMO system, we always sense the preamble of the package included. Taking the characteristics of the preamble into consideration, the duration of single energy detection is adaptive for clear channel access schemes. The algorithm of the energy detection is as shown in Eq. (10).

$$
p(t)=\int_{t-W D}^{t}|r(\tau)|^{2} d_{\tau}
$$

where $p(t)$ is the detected energy at time $t$, wD is the duration of energy detection window and $r()$ is the signal to be detected. Since the duration of energy detection window is limited, the bias of the energy is not small enough especially at condition of lower SNR. If we employ energy detection for channel sensing, the detection sense should at least $3 \mathrm{~dB}$ above noise and with little ability to combat disturb. It is an impossible task to accurately sense the channel if only use energy detection.

The carrier sensing combined with energy detection is an optimum method. The signals exploited by carrier sense in the IEEE 802.11n include DSSS and OFDM short preamble field. The local DSSS sequence exploited in the IEEE

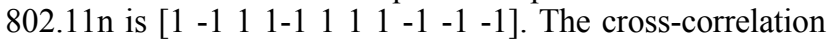
between the received signals and local sequence is employed to sense the received carrier. In order to combat the noise and interference, two thresholds were set. Namely, one is to check the peak average ratio (PAR) and the other is to check the amplitude of the maximum cross-correlation results. This method was proved to be very useful in Section IV. Since the short preamble field exploited in OFDM frame has good auto-correlation characteristics, the auto-correlation is usually employed to sense whether the received signals is the OFDM frame including in IEEE802.11n.

Both energy detection and carrier sense referred above are employed to sense both upper-band and lower-band when the device in $20 \mathrm{MHz} / 40 \mathrm{MHz}$ mode. If the two subbands are sensed busy, the detection results need to be modified by comparing the power of the sub-bands. If the sub-band with stronger power is above $6 \mathrm{~dB}$ more than the weaker one, it should be concluded that only the sub-band with stronger power is busy. Moreover, the results of energy detection are prior to the result of the carrier sense.

\section{Simulation Results}

The functional simulation is under TGn Channel Models [7][8] which are varied typical channel models. The center frequency is set to $5320 \mathrm{MHz}$. The CFO and SCO are both $13.65 \mathrm{ppm}$ according to the TGn comparison criteria. The performances of the proposed methods are shown in Figure 2. The simulation results demonstrate that the proposed method is much better than that only employing energy detection as channel sense. The performances also show that the proposed method make sure that the channel sense is not a roadblock of the IEEE 802.11n system.

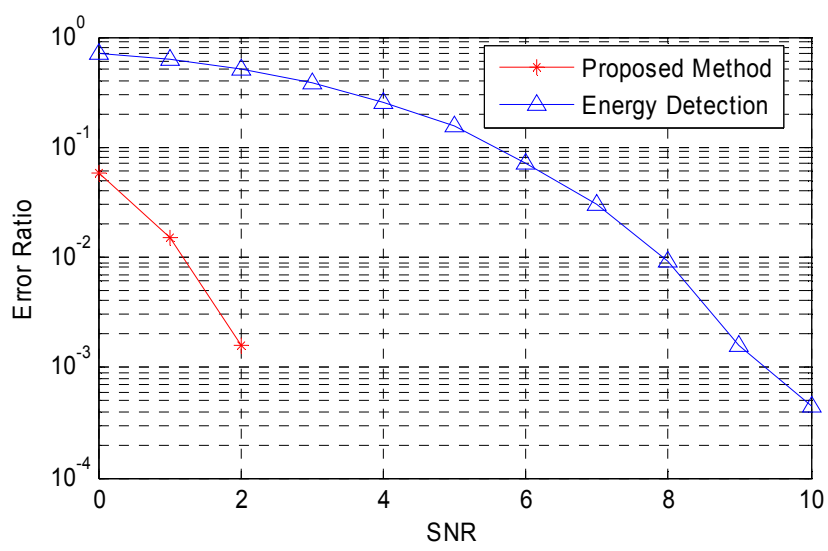

Figure 2. The performance of the proposed method Vs ED method

\section{SUMMARIES}

A novel channel sensing method for IEEE 802.11n systems is proposed. The proposed method is robust to low signal-to-noise ratio (SNR) and highly frequency selective channel. Moreover, this method is self-adaptive and reconfigurable. Therefore, it provides a smart selection for clear channel access (CCA) scheme. The simulation results show that the proposed method provides good performance and high robustness. In general, the proposed method can be the optimum solution for channel sending especially for IEEE 802.11n system.

\section{REFERENCES}

[1] IEEE Std 802.11nTM-2009: Available: http://standards.ieee.org/ getieee802/downl-oad/802.11n-2009.pdf.

[2] Y. Zhu, Q. Zhang, Z. Niu and J. Zhu, On Optimal Physical Carrier Sensing: Theoretical Analysis and Protocol Design, IEEE INFOCOM (2007).

[3] X. Yang and N. H. Vaidya, On Physical Carrier Sensing in Wireless Ad Hoc Network, IEEE INFOCOM (2005).

[4] J. A. Fuemmeler, N. H. Vaidya and V. V. Veeravalli, Selecting Transmit Powers and Carrier Sense Thresholds for CSMA Protocols, UIUC Technical Report, Oct, 2004. 
[5] Eldad Perahia and Robert Stacey. Next Generation Wireless LANs. Cambridge University Press, 2008.

[6] Thomas Paul and Tokunbo Ogunfunmi, Understanding IEEE802.11n Amendment, IEEE Circuits and System Magazine first Quarter2008,28-54
[7] TGn Channel Models, IEEE std. 802.11-03/940r4. may, 2004. Available: http://www.ieee802org/11/DocFiles/03/11-0300940-04000n-tgn-channel-models.doc.

[8] Prof. S. S. Haykin, Ph.D., D.Sc., C.Eng., M.I.E.E Different forms of the Hilbert transform as applied to digital filters and sequence, PROC.IEE, Vol.121, No.7, JULY 1974, pp. 561-567. 